Article

\title{
Analytic Extension of Riemannian Analytic Manifolds and Local Isometries
}

\author{
Vladimir A. Popov \\ Financial University under the Government of the Russian Federation, 125167 Moscow, Russia; \\ vlapopov@gmail.com
}

Received: 21 September 2020; Accepted: 19 October 2020; Published: 22 October 2020

check for updates

\begin{abstract}
This article deals with a locally given Riemannian analytic manifold. One of the main tasks is to define its regular analytic extension in order to generalize the notion of completeness. Such extension is studied for metrics whose Lie algebra of all Killing vector fields has no center. The generalization of completeness for an arbitrary metric is given, too. Another task is to analyze the possibility of extending local isometry to isometry of some manifold. It can be done for metrics whose Lie algebra of all Killing vector fields has no center. For such metrics there exists a manifold on which any Killing vector field generates one parameter group of isometries. We prove the following almost necessary condition under which Lie algebra of all Killing vector fields generates a group of isometries on some manifold. Let $\mathfrak{g}$ be Lie algebra of all Killing vector fields on Riemannian analytic manifold, $\mathfrak{h} \subset \mathfrak{g}$ is its stationary subalgebra, $\mathfrak{z} \subset \mathfrak{g}$ is its center and $[\mathfrak{g}, \mathfrak{g}]$ is commutant. $G$ is Lie group generated by $\mathfrak{g}$ and is subgroup generated by $\mathfrak{h} \subset \mathfrak{g}$. If $\mathfrak{h} \cap(\mathfrak{b}+[\mathfrak{g} ; \mathfrak{g}])=\mathfrak{h} \cap[\mathfrak{g} ; \mathfrak{g}]$, then $H$ is closed in $G$.
\end{abstract}

Keywords: Riemannian analytic manifold; analytic extension; Lie algebra and Lie group; Killing vector field

\section{Introduction}

For a long time, the "curvilinearity" of our space was scientifically substantiated. The geometry of our space does not obey the laws of Euclidean geometry, but is determined by the general concept of the Riemannian metric. So, we can determine the local properties of the surrounding space, but the global structure of the universe as a whole is very difficult to be imagined. The prevailing opinion expressed by A. Poincare is that, by analogy with the surface of the earth, the universe is a closed simply connected space. Poincare put forward a hypothesis according to which a closed, simply connected three-dimensional space is topologically equivalent to a three-dimensional sphere, which leads to some analogy between the structure of the universe and the structure of the earth's surface. Recently, the purely mathematical hypothesis of Poincare was finally proved by G. Perelman.

It is possible to try an analytical approach to the study of global properties of Riemann space. This approach is related to the fact that the Riemann tensor is given by analytic functions that have the property of unique analytic extension. Consider a Riemannian analytic manifold and a ball $U \subset M$ of small radius centered at some point $x_{0} \in M$. By an analytic extension of a locally defined metric, we mean any Riemannian analytic manifold $N$ of the same dimension and an analytic isometry $\varphi: U \rightarrow N$. Let us try to find the most natural analytical extension of this metric. As a natural requirement to analytic extension, the result of extension must be nonextendable. The notion of nonextendable Riemannian analiytic manifold was introduced in the classical monographs of Helgason [1] and S. Kobayashi, Sh. Nomizu [2]. However, nonextendable manifolds can be very unnatural. For example, a simply connected covering of the right half-plane without the points $\left(\frac{1}{n} ; \frac{k}{n}\right)$, $k, n \in \mathbb{N}$. Analytic extension of Riemannian analytic manifolds without Killing vector fields and some spaces of affine connection was studied in the thesis of G.H. Smith [3]. Analytic extension of 
Riemannian analytic manifolds with Killing vector fields and closeness of Lie subgroups is studied in this article.

Generally, the essential requirement for researching in global geometry of Riemannian spaces is the completeness of the manifold under consideration. For a complete simply connected Riemannian analytic manifold $M$, any isometry $\varphi: U \rightarrow V$ between two connected open subsets $U \subset M, V \subset M$ analytically extends to the isometry $\varphi: M \rightarrow M[1]$.

However, usually the ball $U$ of a Riemannian analytic manifold cannot be isometrically embedded in a complete Riemannian analytic manifold, i.e., generally speaking, a locally defined Riemannian metric does not analytically extend to the metric of a complete Riemannian manifold. We have a problem of a generalization of the notion of completeness. A natural generalization of this kind is the nonextendability of a Riemannian analytic manifold. However, nonextendable manifolds can be quite unnatural.

We ask ourselves whether it is possible to construct a Riemannian analytic manifold $M$ containing $U$ as an open subset and admitting analytic extension of local isometries to isometries of the whole manifold using the given local properties of the Riemannian analytic metric, i.e., the metric defined on a small ball $U$. That is, any isometry $\varphi: U \rightarrow V$ between two connected open subsets $U \subset M, V \subset M$ analytically extends to the isometry $\varphi: M \rightarrow M$. The following fact is an insurmountable obstacle to such extension. Let $\mathfrak{g}$ be the Lie algebra of all Killing vector fields on a Riemannian analytic manifold $M$ and $\mathfrak{h} \subset \mathfrak{g}$ its stationary subalgebra for a fixed point $p \in M, X \in \mathfrak{h} \Leftrightarrow X(p)=0$. Let $G$ be the simply connected subgroup generated by the algebra $\mathfrak{g}$, and $H$ be its subgroup generated by the subalgebra $\mathfrak{h}$. Let $G$ act on a simply connected manifold $M$; then the orbit of a fixed point $p \in M$ is a submanifold isometric to the group $G / H$, but the factor group $G / H$ is a manifold if and only if the subgroup $H$ is closed in $G$, and this is not always true.

The aim of this paper is to define a pseudocomplete manifold, which is the "most complete" analytic extension of an arbitrary locally given Riemannian analytic metric. An analytic extension of a locally given Riemannian metric is studied. We consider cases of a completely inhomogeneous metric and metric for which the Lie algebra of all vector Killing fields does not have a center. In these cases, we define a quasicomplete manifold $M$ with the property of uniqueness and extendability of all local isometries $f: U \rightarrow V$, where $U, V$ are connected open subsets of the manifold $M$, up to the isometry $f: M \rightarrow M$. An oriented Riemannian analytic manifold whose vector field algebra has a zero center is called quasicomplete if it is nonextendable and does not admit nontrivial local isometries into themselves, preserving orientation and all Killing vector fields.

We give a definition of a pseudocomplete manifold that leads to the "most complete" extension of a locally given metric and applies to an arbitrary locally given metric. A Riemannian analytic simply connected oriented manifold $M$ is called pseudocomplete if it has the following properties: $M$ is nonextendable. There is no locally isometric orientation preserving covering map $f: M \rightarrow N$, where $N$ is a simply connected Riemannian analytic manifold, and $f(M)$ is an open subset of $N$ not equal to $N$. Among the pseudocomplete manifolds, we distinguish the "most symmetric" regular pseudocomplete manifolds. Next, we study pseudocomplete manifolds of small dimensions and give their classification.

The second goal is to study locally homogeneous manifolds, not only Riemannian, but also pseudo-Riemannian. Below we give the conditions under which $H$ is closed in $G$. The structure of nonclosed subgroups is well known. However, the corresponding studies use the structure of groups $G$ and $H$ and do not take into account the local properties of the Riemannian metric. A description of the properties of an open subgroup $H \subset G$ is contained in the classical work of A. I. Maltsev [4]. If the Lie subgroup $H$ of a simply connected Lie group $G$ is not closed in $G$, then the group $G$ contains a torus $T$ such that the intersection $H \cap T$ is an everywhere dense winding of this torus. However, this fact is difficult to establish on the basis of the local properties of a given Riemannian analytic metric, i.e., on the basis of the properties of the Lie algebra $\mathfrak{g}$ and its stationary subalgebra $\mathfrak{h}$. Is it possible to find the properties of the Lie algebra of all Killing vector fields for which the subgroup $H$ defined 
by the stationary subalgebra $\mathfrak{h}$ is closed in the simply connected group $G$ generated by the algebra g. We should mention the result of Mostow, according to which $H$ is closed in $G$ if $\mathfrak{h}$ is semisimple. In addition, Mostow proved that $H$ is closed in $G$ if $\operatorname{dimg}-\operatorname{dimh}<5$, [5].

Let us try to find the necessary and sufficient properties of the Lie algebra $g$ of all Killing vector fields on the Riemannian analytic manifold $M$ and its stationary subalgebra $\mathfrak{h}$, under which $H$ is closed in $G$. Purely algebraic means are not enough here. To study the closeness problem for a stationary subgroup $H$ in a simply connected group $G$, we use the study of the analytic extension of a locally given Riemannian analytic metric. Manifolds that are analytic extensions of an arbitrary locally given Riemannian analytic metric have the same Lie algebra of all Killing vector fields. Therefore, the question of whether the group $H$ is closed in $G$ is equivalent to the question of the analytic extendability of a locally given Riemannian analytic metric on a locally homogeneous space to the metric of a complete manifold. The concept of an analytic extension of a Riemannian analytic metric was given in the classical monographs of Helgason [1] and S. Kobayashi, Sh. Nomizu [2], but it was not developed. It was developed in [3].

The case when $\mathfrak{g}$ has a zero center was studied in the papers [6-8] not only for Riemannian manifolds, but also for pseudo-Riemannian spaces and spaces with affine connection. It is proved that in this case the subgroup $H$ defined by the stationary subalgebra $\mathfrak{h}$ is closed in the simply connected group $G$ generated by the algebra $\mathfrak{g}$. In addition to the algebraic approach, an analytical approach is being developed to study the analytic extension of Riemannian analytic manifolds. One of the main topic of this paper is the study of locally homogeneous manifolds whose Lie algebra $g$ of all Killing vector fields has a nontrivial center $\mathfrak{z}$. The properties of the algebra $\mathfrak{g}$, its stationary subalgebra $\mathfrak{h}$ and the center $\mathfrak{z}$, which ensures the closedness of the subgroup $H$ defined by the stationary subalgebra $\mathfrak{b}$, in the simply connected group $G$ generated by the algebra $g$ are given. Let $z$ be the center of the algebra $\mathfrak{g}, \mathfrak{r}$ its radical, and $[\mathfrak{g} ; \mathfrak{g}]$ its commutant. If $\mathfrak{h} \cap(\mathfrak{b}+[\mathfrak{g} ; \mathfrak{g}])=\mathfrak{h} \cap[\mathfrak{g} ; \mathfrak{g}]$, then $H$ is closed in $G$. If for any semisimple subalgebra $\mathfrak{p} \subset \mathfrak{g}$ such that $\mathfrak{p}+\mathfrak{r}=\mathfrak{g}$ holds an equality $(\mathfrak{p}+\mathfrak{z}) \cap \mathfrak{h}=\mathfrak{p} \cap \mathfrak{h}$, then $H$ is closed in $G$.

It is of fundamental importance to study the case of a completely inhomogeneous Riemannian metric, i.e., a metric that does not admit any one parameter local group of local isometries (Killing vector fields). In this case, it is possible to define a so-called quasicomplete manifold that has the property of unextendability and uniqueness for each locally given completely inhomogeneous metric, [9]. The definition of a quasicomplete manifold can be generalized to the case when the Lie algebra of all Killing vector fields for a given locally defined Riemannian analytic metric has no center, [8]. Such manifold $M$ has the property of the maximum possible symmetry, i.e., any isometry $f: U \rightarrow V$ $\mathrm{V}$ between connected open subsets of the variety $M$ can be analytically extended to an isometry $f: M \rightarrow M$. However, a quasicomplete manifold has not only the disadvantage that it is not defined for an arbitrary locally given metric, but in a certain sense it is not "the most complete." We present the concept of a pseudocomplete manifold for an arbitrary locally given Riemannian metric, investigate its properties and connection with a quasicomplete manifold, and also describe pseudocomplete manifolds in the case of small dimensions.

\section{Analytic Extension of Riemannian Manifolds and Generalization of the Notion of Completeness}

The class of all locally isometric Riemannian analytic manifolds will also be called the class of manifolds originating from a given germ of a Riemannian analytic manifold, and a specific manifold from this class will be called the analytic extension of this germ. A natural requirement for the analytic extension of a germ is the nonextendability of the resulting manifold. Let us move on to precise definitions and formulations. 
Definition 1. An analytic extension of a Riemannian analytic manifold $M$ is a Riemannian analytic manifold $N$ such that there exists an analytic embedding of $M$ into $N$ as a proper open subset. A manifold that does not admit analytic extension is called nonextendable.

Definition 2. A local isometry between two Riemannian analytic manifolds $M$ and $N$ is the isometry $\varphi: U \rightarrow V$ between open subsets $U \subset M, V \subset N$. The manifolds between which there is a local isometry will be called locally isometric.

Any vector field $X \in \mathfrak{g}$ can be analytically extended along any curve on the manifold $M$ and, thus, the Lie algebra $g$ defines the Lie algebra $g$ of Killing vector fields on any simply connected manifold $N$ locally isometric to $M$. This fact is also true for manifolds with affine connection.

Lemma 1. Let $M$ be an analytic manifold with affine connection, $X$ an infinitesimal affine transformation defined in a domain $U \subset M$ and let $\gamma(t), 0 \leq t \leq 1$, be a continuous curve in $M$ such that $\gamma(0) \in U$. Then the vector field $X$ is analytically extendable along $\gamma$. If the curves $\gamma(t)$ and $\delta(t), 0 \leq t \leq 1, \gamma(0)=\delta(0), \gamma(1)=\delta(1)=x_{1}$, are homotopic, then the extension of the vector field $X$ to the point $x_{1}$ along these curves coincide.

Proof. Suppose that $X$ is analytically extendable to a neighborhood of any point $\gamma(t)$ for $0 \leq \mathrm{t}<t_{1}<1$. Let us prove that it also extends to a neighborhood of the point $q=\gamma\left(t_{1}\right)$. Let $V$ be a normal neighborhood of a point $q$, which is a normal neighborhood of each of its points [1]. Consider $\mathrm{t} \leq t_{1}$ such that $p=\gamma(t) \in V$.

The vector field $X$ generates a local one-parameter isometry group $\varphi_{s}$ in a neighborhood of each point $\gamma(t), \mathrm{t}<t_{1}$. Let us prove that for all sufficiently small values of $s$, the local isometries $\varphi_{s}$ analytically extend to a neighborhood of the point $=\gamma\left(t_{1}\right)$. Then the tangent vector field of this local group of isometries will be the analytic extension of the vector field $X$ to a neighborhood of the point $q$.

Let us consider a connected open set $V_{0}$ containing points $p$ and $q$, whose closure also belongs to $V, \bar{V}_{0} \subset V, p, q \in V_{0}$. Let us consider a small neighborhood $V^{\prime} \subset V_{0}$ of the point $q$ and connect the point $p$ with a segment of the geodesic $\alpha(t), 0 \leq t \leq 1$, with an arbitrary point $q^{\prime} \in V^{\prime}$. Let $Y=\frac{d \alpha}{d t}(0) \in T_{p} M$ and $p_{s}=\varphi_{s}(p), Y_{s}=\varphi_{s}(Y)$. From the point $\beta(t), 0 \leq t \leq 1$, we draw a geodesic $\beta(t), 0 \leq t \leq 1$ such that $\frac{d \beta}{d t}(0)=Y_{s}$. For sufficiently small values of $\beta(t) \in V_{0}, 0 \leq t \leq 1$. We put $\varphi_{s}\left(q^{\prime}\right)=\beta(1)$. The mapping thus obtained is the analytic extension of the isometry $\varphi_{s}$.

Let us study the case of a completely inhomogeneous Riemannian metric, i.e., a manifold with such metric that has not any Killing fields. In this case, it is possible to define a quasicomplete variety that has the property of nonextendability and uniqueness for each locally given completely inhomogeneous metric [8].

Definition 3. An analytic Riemannian manifold is called a completely inhomogeneous manifold if there are no Killing vector fields on it. The Riemannian metric of a completely inhomogeneous manifold is called a completely inhomogeneous metric.

By Lemma 1, all manifolds that are locally isometric to a completely inhomogeneous manifold are completely inhomogeneous.

Definition 4. A completely inhomogeneous oriented Riemannian analytic manifold is said to be quasicomplete if it is nonextendable and does not admit nontrivial orientation-preserving local isometries into itself.

Let us present the main properties of completely inhomogeneous quasicomplete manifolds, [9]. For an arbitrary completely inhomogeneous manifold $M$, consider the set $S \subset M$ of all fixed points of all possible orientation-preserving local isometries of the manifold $M$ into itself. 
Theorem 1. For an arbitrary completely inhomogeneous Riemannian analytic manifold $M^{\prime}$, the set $S \subset M^{\prime}$ is an analytic subset of codimension at least 2. Consequently, $M^{\prime} \backslash S$ is a connected manifold.

Theorem 2. For any completely inhomogeneous Riemannian analytic manifold $M^{\prime}$ there exists a quasicomplete manifold $M$ locally isometric to it and a locally isometric covering map $f: M^{\prime} \backslash S \rightarrow M$. Thus, a quasicomplete manifold is unique for every completely inhomogeneous locally given Riemannian analytic metric.

The proof of Theorems 1 and 2 can be found in [9].

The definition of a quasicomplete manifold can be generalized to the case when the Lie algebra of all Killing vector fields for a given locally defined Riemannian analytic metric has no center, [8]. Such are also locally homogeneous manifolds, in particular all locally symmetric spaces.

Definition 5. An analytic Riemannian manifold $M$ is called to be locally homogeneous if at any point $p \in M$ the Killing vector fields form a basis of the tangent space $T_{p} M$.

An equivalent definition of a locally homogeneous manifold $M$ is that for any points $p, q \in M$ there exists a local isometry $\varphi$ of the manifold $M$ such that $\varphi(p)=q$.

Definition 6. An oriented Riemannian analytic manifold whose algebra Lie of all Killing vector fields has no center is called quasicomplete if it is nonextendable and does not admit nontrivial preserving the orientation and all Killing vector fields of local isometries into itself.

Let us investigate oriented Riemannian analytic manifolds whose Lie algebra of all Killing vector fields has no center in order to prove that each such manifold is locally isometric to a quasicomplete manifold, and a locally homogeneous quasicomplete manifold is a complete homogeneous manifold.

We denote by $Z(M)$, the pseudogroup of all local isometries of a Riemannian analytic manifold $M$ preserving all Killing vector fields and orientation, $\varphi \in Z(M) \Leftrightarrow \forall X \in \mathfrak{g} \varphi(X)=X$.

Lemma 2. Let $M$ be an analytic Riemannian manifold satisfying the property of unique analiytic extension of Killing vector fields and whose Lie algebra of all Killing vector fields has no center. Then the set $S \subset M$, consisting of fixed points of all possible isometries $\varphi \in Z(M)$, is an analytic subset of codimension at least 2.

Proof. Let us prove that for any open set $U \subset M$ with compact closure there are only finitely many local isometries from $U$ to $U$ belonging to the pseudogroup $Z(M)$. Assume the opposite and consider an infinite sequence of local isometries $\varphi_{i}$ from $U$ to $U, \varphi_{i} \in Z(M)$. In the proof of Lemma 3 in [8], was constructed a Killing vector field $X$ on some open set $V \subset U$ which satisfies the following condition. For some subsequence of local isometries $\varphi_{i} \forall t,|t| \leq 1, \forall i \in \mathbb{N}, \exists k(i) \in \mathbb{N}$ such that $\lim _{i \rightarrow \infty} \varphi_{i}^{k(i)}=\operatorname{ExptX}$, where Expt $X$ is the local one-parameter isometry group generated by the vector field $X$. Therefore,

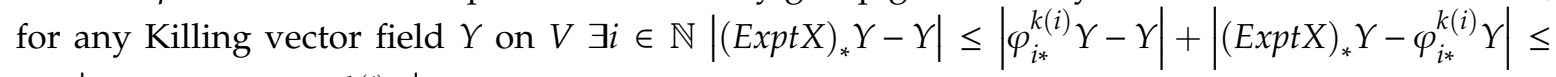
$0+\left|Y-(\operatorname{Exp}(-t X)) \varphi_{i *}^{k(i)} Y\right| \leq \frac{1}{2}\left|(\operatorname{ExptX})_{*} Y-Y\right|$. Therefore $\forall Y \in \mathfrak{g}(\operatorname{ExptX})_{*} Y=Y$, i.e. $[X, Y]=0$. It contradicts to the absence of center in $\mathfrak{g}$.

This contradiction proves the existence of only finite number of local isometries from $U$ to $U$ belonging to the pseudogroup $Z(M)$. As it was shown in [8], it easily follows from it that the set $S$ is an analytic subset of codimension not less than 2.

By Lemma 2, the manifold $M \backslash S$ is connected.

Lemma 3. Let $M$ be an analytic Riemannian manifold satisfying the property of unique extension of Killing vector fields and whose Lie algebra of all Killing vector fields has no center. Then there is a locally isometric covering map from $M \backslash S$ to an analytic Riemannian manifold $M_{1}$, which also satisfies the property of unique extension of Killing vector fields, and whose pseudogroup $\mathrm{Z}\left(\mathrm{M}_{1}\right)$ consists only of the identity transformation. 
Proof. Let us factorize the variety $M \backslash S$ by the pseudogroup $Z(M)$. It follows from the proof of Lemma 2 that for every point $x \in M \backslash S$ there is a neighborhood $U_{1 x} \subset M \backslash S$ of the point $x$ that does not admit nonidentical orientation-preserving local isometries from $U_{1 x}$ to $U_{1 x}$ belonging to the pseudogroup $Z(M)$. This proves that the quotient map $\pi$ projecting the manifold $M \backslash S$ into the set $M_{1}=M \backslash S / Z(M)$ is a covering map. Hence, for each point $x \in M$, there is a neighborhood $U_{x} \subset M_{1}$ of it and an open set $V_{x} \subset \pi^{-1}\left(U_{x}\right)$, such that the mapping $\pi$ establishes a homeomorphism between the sets $V_{x}$ and $U_{x}$. Let us define a Riemannian scalar product. If necessary, going to subset of the set $V_{x} \subset M \backslash S$, we will assume that $V_{x}$ is a coordinate neighborhood of the point $y \in \pi^{-1}\left(U_{x}\right) \subset M \backslash S$. Then we declare the set $U_{x} \subset M_{1}$ as a coordinate neighborhood of the point $x \in M_{1}$. Consider two such neighborhoods $U_{1}, U_{2} \subset M_{1}, U_{1} \cap U_{2} \neq \varnothing$. Let the sets $V_{1}, V_{2} \subset M \backslash S$ corresponds to the sets $U_{1}, U_{2}$. Put $\pi^{-1}\left(U_{1} \cap U_{2}\right) \cap V_{1}=V_{10}, \pi^{-1}\left(U_{1} \cap U_{2}\right) \cap V_{2}=V_{20}$. Then there is an isometry $\alpha: V_{10} \approx V_{20}$. Let $\psi_{1}$ and $\psi_{2}$ be coordinate mappings on $V_{1}$ and $V_{2}$, respectively. Then $\psi_{1} \pi^{-1}$ and $\psi_{2} \pi^{-1}$ will be coordinate maps to $U_{1}$ and $U_{2}$.

Let us consider an arbitrary point $x \in M_{1}$ and arbitrary vectors $X, Y \in T_{x} M_{1}$. Let us also consider some point $y \in \pi^{-1}(x) \subset M \backslash S$ and vectors $X_{1}, Y_{1} \in T_{y} M$ such that $\pi_{*} X_{1}=X, \pi_{*} Y_{1}=Y$. Let us define the Riemannian scalar product $\langle X, Y\rangle$ equal to the Riemannian scalar product $\left\langle X_{1}, Y_{1}\right\rangle$ on $T_{x} M$. If we take another point $z \in \pi^{-1}(x)$ and vectors $X_{2}, Y_{2} \in T_{z} M$ such that $\pi_{*} X_{2}=X, \pi_{*} Y_{2}=Y$, then there exists a local isometry $\varphi \in Z(M)$ such that that $\varphi(z)=y, \varphi_{*} X_{2}=X_{1}, \varphi_{*} Y_{2}=Y_{1}$. Therefore, $\left\langle X_{1}, Y_{1}\right\rangle=\left\langle X_{2}, Y_{2}\right\rangle$. This proves the correctness of the definition of the Riemannian metric on $M_{1}$.

The constructed Riemannian manifold $M_{1}$ does not admit nonidentical orientation-preserving local isometries that induce the identity transformation on the algebra of Killing vector fields $\mathfrak{g}$. The projection $\pi: M \backslash S \rightarrow M_{1}$ is a locally isometric covering map. It remains to prove the property of unique extension of Killing vector fields to $M_{1}$. Consider a Killing vector field $X$ defined on some open set $U \subset M_{1}$ and open sets $U_{0} \subset U$ and $V_{0} \subset M \backslash S$ such that the covering map $\pi$ establishes an isometry between the sets $V_{0}$ and $U_{0}$. Then the vector field $\pi_{*}^{-1} X$ extends uniquely from the set $V_{0} \subset M$ to the whole manifold $M$ and defines a vector field $Y$ on $M$. Let points $y, z \in M \backslash S$ be such that $\pi(\mathrm{x})$ $=\pi(\mathrm{y})$ and $\pi_{*} Y(z)=\pi_{*} \varphi_{*} Y(y)$. Since $\pi \cdot \varphi=\pi$ by the definition of $\pi$, then $\pi_{*} \cdot \varphi_{*}=\pi_{*}$. Therefore, $\pi_{*} Y(z)=\pi_{*} \varphi_{*} Y(y)=\pi_{*} Y(y)$. This proves that the mapping $\pi$ uniquely projects the vector field $Y$, given on $M$, onto the vector field $\pi_{*} Y$, given on the manifold $M_{1}$. The resulting vector field $\pi_{*} Y$ will be the analytic extension of the vector field $X$ to the entire manifold $M_{1}$.

Theorem 3. An arbitrary Riemannian analytic manifold $M$ whose Lie algebra of Killing vector fields has no center is locally isometric to a quasicomplete manifold.

Proof. Let us consider an arbitrary Riemannian analytic manifold $M^{\prime}$, the Lie algebra of Killing vector fields of which has no center and construct for it the manifold $M_{1}^{\prime}$ similarly to the construction of the manifold $M_{1}$ in the proof of Lemma 3. $M_{1}^{\prime}$ does not admit local isometries in itself that preserve the orientation and vector Killing fields. Then a quasicomplete manifold $M$ will be some maximal analytic extension of the manifold $M_{1}^{\prime}$. We will assume that all the manifolds that we will consider in the proof of the theorem have the property of unique analytic extension of Killing vector fields, that is, the Lie algebra of all Killing vector fields is the same for all manifolds and is equal to $\mathfrak{g}$. If $M^{\prime}$ satisfies this property, then the manifold $M_{1}^{\prime}$ also satisfies it.

Let us consider the set $\Lambda$ consisting of analytic extensions $M_{\alpha}$ of the manifold $M_{1}$ that satisfy the property of unique extension of Killing vector fields and do not admit local isometries that are identical on the algebra of all Killing vector fields. We mark a point on a manifold $M_{1}$ and a basis of tangent space at this marked point, and also mark the images of this point and this basis in the manifolds $M_{\alpha} \in \Lambda$. Let us introduce the following order relation on this set. $M_{\alpha} \leq M_{\beta}$ if there is an isometric embedding $i_{\alpha \beta}: M_{\alpha} \rightarrow M_{\beta}$ which transfers the marked point to the marked and marked basis of the tangent space to the marked one. As a result, $\Lambda$ becomes a partially ordered set. Consider an arbitrary linearly ordered subset $\Delta$ of the set $\Lambda$. Let us construct the direct limit of the family of 
manifolds $M_{\alpha} \in \Delta$ and mappings $i_{\alpha \beta}$. We obtain a manifold $M_{0}$ with the following properties. For any manifold $M_{\alpha} \in \Delta$ there is an isometric embedding $i_{\alpha}: M_{\alpha} \rightarrow M_{0}$ and $i_{\alpha}\left(M_{\alpha}\right) \subset i_{\beta}\left(M_{\beta}\right)$, if $M_{\alpha} \leq M_{\beta}$. $M_{0}=\cup_{M_{\alpha} \in \Delta}\left(M_{\alpha}\right)$. Let us prove that $M_{0} \in \Lambda$. An arbitrary vector field $X$ on the manifold $M_{1}$ with the help of the imbeddings $i_{1 \alpha}: M_{1} \rightarrow M_{\alpha}$ and $i_{\alpha}: M_{\alpha} \rightarrow M_{0}$ is transferred to the manifold $i_{\alpha}\left(M_{\alpha}\right) \subset M_{0}$, moreover, $\left(i_{\alpha} \cdot i_{1 \alpha}\right)_{*} X=\left(i_{\beta} \cdot i_{1}\right)_{*} X$ to $i_{\alpha}\left(M_{\alpha}\right) \cap i_{\beta}\left(M_{\beta}\right)$ and the Killing vector field $\left(i_{\alpha} \cdot i_{1 \alpha}\right)_{*} X$ uniquely extends from the submanifold $i_{\alpha}\left(M_{\alpha}\right) \subset M_{0}$ to any submanifold $i_{\beta}\left(M_{\beta}\right) \subset M_{0}, M_{\beta} \geq M_{\alpha}$ and therefore, to the whole manifold $M_{0}$. Thus, a Killing vector field defined on an arbitrarily small open set $U \subset M_{0}$ can be uniquely extended to a Killing vector field on $M_{0}$.

Let us consider now a local isometry $\varphi \in Z\left(M_{0}\right)$. Let the point $x_{0} \in M_{0}$ belong to the domain of the isometry $\varphi$. Then the points $x_{0}$ and $\varphi\left(x_{0}\right)$ lie in some submanifold $i_{\alpha}\left(M_{\alpha}\right) \subset M_{0}$. Therefore, $\varphi \in Z\left(i_{\alpha}\left(M_{\alpha}\right)\right)$ and therefore $\varphi$ is the identity transformation. Hence, the pseudogroup $Z\left(M_{0}\right)$ consists only of the identity transformation. For an arbitrary linearly ordered subset $\Delta \subset \Lambda$ we have constructed the supremum. By Zorn's lemma, the set $\Lambda$ has a maximal element. We assert that the manifold $M$, which is a maximal element, is the required quasicomplete manifold. It is required to prove that $M$ is not extendable.

Suppose the opposite and denote by $N$ a nontrivial extension of the manifold $M$. Let $S \subset N$ as above denote the set of fixed points of all possible local isometries from the pseudogroup $Z(N)$. Let us factorize the manifold the manifold $N \backslash S$ in exactly the same way as in the proof of Lemma 3, the way manifold $M \backslash S$ was factorized. As a result, we obtain a manifold $L$ that satisfies the property of unique extension of Killing vector fields and does not admit local isometries that preserve the orientation and all Killing vector fields. Let $i$ denote the embedding $i: M \rightarrow N$. Let us prove that $i(M) \cap S=\varnothing$. If $x \in i(M)$ then some normal ball $B$ centered at $x$ belongs to $i(M)$. If, in addition $x \in S$ then there is a local isometry $\varphi \in Z(M)$ satisfying the condition $\varphi(x)=x$. This isometry defines the isometry of the ball $B$ into itself, given in normal coordinates by a linear map-the differential of isometry $\varphi$, but the existence of such an isometry contradicts the triviality of the pseudogroup $Z(M)$. Thus, $i$ gives an embedding $i: M \rightarrow N \backslash S$. The chain mapping $\pi \rightarrow i: M \rightarrow L$ where $\pi: N \backslash S \rightarrow L$ is the covering mapping constructed in the proof of Lemma 3 and is also an embedding. Since, if $\pi \cdot i(x)=\pi \cdot i(y)$ then there is a local isometry $\varphi \in Z(M)$ such that $\varphi(x)=\varphi(y)$, hence $x=y$. Since $M$ is a maximal element of the set $\Lambda, \pi \cdot i$ is an isometry, and $N \backslash S$ covers $M$.

We have a covering map $\pi: N \backslash S \rightarrow M$ and an embedding $i: M \rightarrow N \backslash S$ where $i(M)$ is open subset in $N \backslash S$. Let there be a sequence of points $x_{n} \in i(M)$ converging to $x \in N \backslash S$. Then the sequence $y_{n}=\pi\left(x_{n}\right)$ also converges to some point $y \in M$. Since $x_{n}=i\left(y_{n}\right)$ then $x=i(y) \in i(M)$. This proves that $i(M)$ is closed in $N \backslash S$. So $N \backslash S$ is disconnected or $N \backslash S=M$, but the disconnectedness of $N \backslash S$ contradicts Lemma 2. Therefore $N \backslash S=M$. Let us prove that $S=\varnothing$. Suppose the opposite and consider a normal ball $B$ centered at some point $x \in S \subset N$. There is nontrivial isometry of the ball $B$ into itself. This isometry does not leave fixed points from $B \backslash S$ and, therefore, is a nonidentical local isometry from the pseudogroup $Z(N \backslash S)$, but since $N \backslash S=M$ this contradicts the triviality of the pseudogroup $Z(M)$. This proves that $S=\varnothing, N=M$ and $M$ is nonextendable.

Theorem 4. Let $\varphi$ be a local isometry from a quasicomplete manifold $M$ to a quasicomplete manifold $N$. Then $\varphi$ extends to an isometry $\varphi: M \approx N$.

Proof. Let us consider an arbitrary point $x \in M$ and a smooth curve $\gamma(t), 0 \leq t \leq 1, \gamma(0) \in D(\varphi) \subset M$, $\gamma(1)=x$. Let us prove that the isometry $\varphi$ defined in the neighborhood $U=D(\varphi) \subset M$ of the point $x_{0}=\gamma(0)$ can be extended along the curve $\gamma$. Suppose there is no such extension. Let number $t_{1} \in[0 ; 1]$ be the minimum among the numbers $t$ such that the isometry $\varphi$ does not extend to a neighborhood of the point $\gamma(t)$ along the curve $\gamma$. Let us prove, nevertheless, that, contrary to the assumption, there exists an extension of $\varphi$ to some neighborhood of the point $\gamma\left(t_{1}\right)$ along the curve $\gamma$.

By the assumption made about $t_{1} \forall t \in\left[0 ; t_{1}\right)$ the isometry $\varphi$ is defined in some neighborhood of the point $\gamma(t)$. So the curve $\delta(t)=\varphi(\gamma(t)), 0 \leq t \leq t_{1}$, is defined on $N$. Let $x_{1}=\gamma\left(t_{1}\right)$ and 
$\varepsilon>0$ be such that the neighborhood $U_{\varepsilon}=\left\{x \in M, \rho\left(x ; x_{1}\right)<\varepsilon\right\}$ is a normal neighborhood of each of its points. Since $\forall y \in N \forall \varepsilon_{0}>0 \exists \alpha$ such that $\forall t^{\prime}, t^{\prime \prime} \in\left[0 ; t_{1}\right)$ under the condition $\left|t_{1}-t^{\prime}\right|<\alpha,\left|t_{1}-t^{\prime \prime}\right|<\alpha$ the following inequalities hold: $\left|\rho\left(y ; \delta\left(t^{\prime}\right)\right)-\rho\left(y ; \delta\left(t^{\prime \prime}\right)\right)\right| \leq \rho\left(\delta\left(t^{\prime}\right) ; \delta\left(t^{\prime \prime}\right)\right) \leq$ $\int_{t^{\prime}}^{t^{\prime \prime}} \sqrt{\left\langle\delta^{\prime}(t) ; \delta^{\prime}(t)\right\rangle} d t=\int_{t^{\prime}}^{t^{\prime \prime}} \sqrt{\left\langle\gamma^{\prime}(t) ; \gamma^{\prime}(t)\right\rangle} d t<\varepsilon_{0}$. Hence $\forall y \in N$ there exists $\lim _{t \rightarrow t_{1}} \rho(y ; \delta(t))=\rho_{1}(y)$. Let us consider the set $V_{\varepsilon}=\left\{y \in N \mid \rho_{1}(y)<\varepsilon\right\}$. There is an isometry $\psi=\varphi^{-1}$ of some neighborhood $V_{D} \subset V_{\varepsilon}$ of the set $D=\left\{y \in N \mid y=\delta(t), t_{2} \leq t<t_{1}\right\}$ to the neighborhood $U_{D} \subset U_{\varepsilon}$ of the set $B=\left\{x \in M \mid x=\gamma(t), t_{2} \leq t<t_{1}\right\}$. Let us prove that $\psi$ can be extended to isometry $\psi: V_{\varepsilon} \approx U_{\varepsilon}$. Let us prove first that $\psi$ can be extended along any curve $v(s), 0 \leq \mathrm{s} \leq 1$ on $V_{\varepsilon}, v(0) \in V_{D}, v(1)=y$ is an arbitrary point on $V_{\varepsilon}$. If we assume that this is not the case, then there is a minimum number $s_{1}$ among the numbers $u \in[0 ; 1]$ with the property: $\psi$ does not extend along the curve $v(s)$ to some neighborhood of the point $v(u)$. Let $\sigma>0$ and $s_{2}<s_{1}$ be such that the set $B_{\sigma}=\left\{y \in N \mid \rho\left(y ; v\left(s_{2}\right)\right)<\sigma\right\}$ is a normal neighborhood of the point $v\left(s_{2}\right)$ and $\rho\left(v\left(s_{2}\right) ; v\left(s_{1}\right)\right)<\frac{\sigma}{2}$. Therefore, $v\left(s_{1}\right) \in B_{\sigma}$. Using the linearity of the map $\psi$ in normal coordinates, one can extend the isometry $\psi$ defined on some neighborhood of the point $v\left(s_{2}\right)$ to the isometry $\psi$ defined on the whole set $B_{\sigma}$ which is a neighborhood of the point $v\left(s_{1}\right)$. This refutes the assumption that $\psi$ cannot be extended along the curve $v(s)$.

Let us prove now that the extension of the isometry $\psi$ to all possible curves to $V_{\varepsilon}$ gives a map $\psi: V_{\varepsilon} \rightarrow U_{\varepsilon}$. Suppose the opposite. Then there is a closed curve $v(t), 0 \leq t \leq 1, v(0)=v(1)$, on $V_{\varepsilon}$ such that the curve $\beta(t)=\psi(v(t))$ on $U_{\varepsilon}$ will be nonclosed, $\beta(0) \neq \beta(1)$. But since all possible analytic extensions of the isometry $\psi$ induce the same maps on the algebra of Killing vector fields, the isometry of the form $\psi \cdot \psi^{-1}$ that maps $\beta(0)$ to $\beta(1)$ belongs to the pseudogroup $Z(M)$ and this contradicts the fact that $M$ is a quasicomplete manifold. Similarly, it is proved that the extension of the local isometry $\varphi=\psi^{-1}$ from $U_{\varepsilon}$ to $V_{\varepsilon}$ defines a map on the $\operatorname{set} \varphi\left(V_{\varepsilon}\right) \subset U_{\varepsilon}$. So, we have an isometric embedding $\psi: V_{\varepsilon} \rightarrow U_{\varepsilon}$. Let us prove that it is a surjective mapping. If we assume the opposite, then gluing the manifolds $N$ and $U_{\varepsilon}$ using the map $\psi$, we obtain a nontrivial extension of the manifold $N$, which contradicts its nonextendability. Therefore, we have an isometry $\psi: V_{\varepsilon} \rightarrow U_{\varepsilon}$. The inverse isometry $\psi^{-1}: U_{\varepsilon} \rightarrow V_{\varepsilon}$ gives an extension of the isometry $\varphi$ to the neighborhood $U_{\varepsilon}$ of the point $\gamma\left(t_{1}\right)$ along the curve $\gamma$, contrary to the initial assumption about $t_{1}$.

Thus, we have proved that the local isometry $\varphi$ from $M$ to $N$ extends to any point $x \in M$ along an arbitrary curve on $M$. Just as above, we proved that the extension of the isometry $\psi$ along all possible curves to $V_{\varepsilon}$ yields a one-to-one mapping defined on the whole $V$, it can be proved that the extension of $\varphi$ along all possible curves to M gives an isometric embedding $\varphi: M \rightarrow N$.

Corollary 1. An arbitrary Riemannian analytic manifold whose Lie algebra of all Killing vector fields has no center is locally isometric to a unique quasicomplete manifold. That is, a locally given Riemannian analytic metric whose Lie algebra of Killing vector fields has no center can be uniquely extended to a quasicomplete manifold.

Proof. Let a quasicomplete manifold $M$ be locally isometric to the manifold $M^{\prime}$ and let $N$ be another quasicomplete manifold locally isometric to the manifold $M^{\prime}$. Then there is a local isometry $\varphi$ from $N$ to $M^{\prime}$ and a local isometry $\psi$ from $M^{\prime}$ to $M$. The superposition of the isometry $\varphi$ and $\psi$ is a locally isometry from $N$ to $M$. By Theorem 4 , the local isometry $\psi \cdot \varphi$ extends to the isometry $M \approx N$.

Corollary 2. Let $\mathfrak{g}$ be the Lie algebra of all Killing vector fields in a Riemannian analytic manifold $M^{\prime}$ diffeomorphic to a ball, and let $\mathfrak{h}$ be its stationary subalgebra. Let $G$ be a simply connected group generated by the algebra $\mathfrak{g}$ and $H$ its subgroup generated by the subalgebra $\mathfrak{h}$. If $\mathfrak{g}$ has no center, then $H$ is closed in $G$.

Proof. Since $M^{\prime}$ is diffeomorphic to a ball, its Killing vector fields can be uniquely analytically extended on it. By Theorem 3, the manifold $M^{\prime}$ is locally isomorphic to a quasicomplete manifold $M$ having the same Lie algebra $\mathfrak{g}$ of all Killing vector fields and the same stationary subalgebra $\mathfrak{h}$. For an arbitrary vector field $X \in \mathfrak{g}$, for all values of the parameter $t$ less than a certain number $\delta$, the elements of the 
one-parameter transformation group ExptX are local isometries of $M$. By Theorem 4, they extend to isometries of the entire manifold $M$. But then the isometries $\operatorname{Exp} n t X=(\operatorname{ExptX})^{n}$ are defined. Thus, the group $G$ acts on $M$, and $H$ is its stationary subgroup. This means that the orbit of the group $G$ on $M$ is covered by the homogeneous manifold $G / H$. Consequently, $H$ is closed in $G$.

Note that quasicomplete manifolds are the most compressed, that is, universally attracting objects in the category of all locally isometric manifolds. For any analytic Riemannian manifold $M^{\prime}$, the algebra of Killing vector fields of which has no center, there exists a locally isometric map from $M^{\prime} \backslash S^{\prime}$ 'to a quasicomplete manifold $M$ defined on the whole $M^{\prime} \backslash S^{\prime}$, where $S^{\prime}$ Is the set of fixed points of all orientation-preserving and Killing vector fields of local isometries of the manifold $M^{\prime}$.

A quasicomplete manifold is unique in the class of all analytic extensions of a given germ and has remarkable properties [9]. First of all, it has the property of maximum symmetry, that is, any local isometry $f: U \rightarrow V$ from a quasicomplete manifold $M$ into itself can be analytically extended to an isometry $f: M \rightarrow M$. However, the concept of a quasicomplete manifold has not only the disadvantage that it is not defined for all locally given Riemannian analytic metrics, but it is also not, in a certain sense, "the most complete." Namely, there is a germ of a Riemannian analytic manifold that can be extended to a complete manifold, the canonical extension of which to a quasicomplete manifold is not a complete manifold.

Example 1. Let us consider an ellipsoid in three-dimensional space, given by the equation $\frac{x^{2}}{a^{2}}+\frac{y^{2}}{b^{2}}+\frac{z^{2}}{c^{2}}=1$. In order to obtain a quasicomplete manifold in the class of all Riemannian analytic manifolds locally isometric to an ellipsoid, it is necessary to remove 6 points of intersection with the coordinate axes from the ellipsoid and factor, with a resulting manifold of the group of rotations by 180 degrees around all coordinate axes.

Nonetheless, it turns out to be possible to give a generalization of the notion of completeness, which leads to the "most complete" manifold for an arbitrary germ of an analytic Riemannian manifold.

Definition 7. A Riemannian analytic simply connected manifold $M$ is called pseudocomplete if it has the following properties.

$M$ is nonextendable.

There is no locally isometric covering map $f ; M \rightarrow N$, where $N$ is a simply connected Riemannian analytic manifold and $f(M)$ is an open subset of $N$ that is not equal to $N$.

Let us investigate the analytic extension to a pseudocomplete manifold for various classes of germs of analytic Riemannian manifolds. First of all, one should establish the fact that an analytic extension to a pseudocomplete manifold exists for any germ of an analytic Riemannian manifold. At the same time, in the general case, this extension is not unique, however, different analytic extensions of the same germ do not differ very significantly.

Theorem 5. Any locally given Riemannian analytic manifold admits an analytic extension to a pseudocomplete manifold. If there is a complete manifold in the class of locally isometric Riemannian analytic manifolds, then this manifold is the only pseudocomplete manifold in this class.

Proof. On the set of all simply connected analytic extensions of a given germ of an analytic Riemannian manifold, we introduce the following order relation. A manifold $M$ is greater than or equal to a manifold $N, M \geqslant N$, if there exists a locally isometric map $f ; N \rightarrow M$. Thus, the set of simply connected Riemannian analytic manifolds that are locally isometric to each other becomes a partially ordered set. By Zorn's lemma, this set contains a maximal element. By definition, this element will be a pseudo-complete manifold.

Let us consider a complete Riemannian analytic manifold $M$. If we assume that $M$ is not pseudocomplete, then there exists a locally isometric map $f ; M \rightarrow N$ such that some point $x \in N$, 
$x \notin f(M)$. Let $\gamma(t) .0 \leq t \leq 1$, be a geodesic connecting point $y \in f(M)$ with point $x$. Then the preimage of this geodesic for $0 \leq t \leq \delta$ does not extend to a geodesic for all $\mathrm{t}$ on the manifold $\mathrm{M}$, which contradicts the completeness of this manifold.

A pseudo-complete manifold is not unique in the class of all locally isometric Riemannian analytic manifolds.

Example 2. Let us consider a germ $\mathcal{A}$ of a two-dimensional analytic Riemannian manifold represented by a sphere with metric $d s^{2}=\frac{f(z, \bar{z})}{\sqrt{1+|z|^{6}}} d z d \bar{z}$, where $f(z, \bar{z})$ is an analytic function on the sphere satisfying the condition $f(z, \bar{z}) \neq\left|A^{\prime}(z)\right|^{2} f(A(z), A(\bar{z}))$ for any linear fractional transformation $A(z)$.

Such a metric has a singularity at the point $z=\infty$. A sphere with this metric is a pseudocomplete manifold. Eliminate the singularity at the point $z=\infty$ using the transformation $z=w^{2}+a$, $a \in \mathbb{C}$. As a result, we obtain a sphere that is double covering the original one and has the metric $d s^{2}=\frac{4|w| f\left(w^{2}+a, \bar{w}^{2}+\bar{a}\right)}{\sqrt{1+\left|w^{2}+a\right|^{2}}} d w d \bar{w}$. This metric has a singularity at the point $w=0$ which is natural, since the sphere $\mathrm{w}$ branches over the sphere $z$ at the point $z=a$ corresponding to the point $w=0$. For different a. we obtain different pseudocomplete manifolds with coordinate $w$.

Example 2 shows that there are many not very natural pseudocomplete manifolds. In order to avoid such unnatural irregularities we will define regular pseudocomplete manifold.

Definition 8. A Riemannian analytic simply connected manifold $M$ is called a regular pseudocomplete manifold if there is no covering locally isometric map $f: M \backslash S \rightarrow N$ into another pseudocomplete manifold $N$ locally isometric to the manifold $M$.

Theorem 6. A local isometry from a regular pseudocomplete manifold $M$ to a regular pseudocomplete manifold $N$ extends analytically along continuous curves to any point $M$ except for an analytic subset $S$ of codimension at least 2.

Proof. We present the proof for the case when the Lie algebra of all Killing vector fields has no center. Consider the subsets $S \subset M$ and $S^{\prime} \subset N$ consisting of all fixed points of local isometries preserving the orientation of Killing vector fields. The sets $S$ and $S^{\prime}$ are analytic subsets of the manifolds $M$ and $N$ of codimension at least $2,[8,9]$. Let $M_{0}$ be a quasicomplete manifold locally isometric to the varieties $M$ and $N$. Then there exist covering locally isometric mappings $f: M \backslash S \rightarrow M_{0}$. and $g: N \backslash S^{\prime} \rightarrow M_{0}[8,9]$. Moreover, it follows from the definition of a regular pseudocomplete manifold that $f(M \backslash S)=M_{0}$ and $g\left(N \backslash S^{\prime}\right)=M_{0}$. Let us consider an arbitrary curve $\gamma(t) \subset M \backslash S$ such that domain of the initially given local isometry $\varphi$ between the manifolds $M$ and $N$ contains the point $\gamma(0)$, its image $\delta(t)=f(\gamma(t)) \subset M_{0}$. and the connected component $\beta(t)$ of the inverse image $g^{-1}(\delta(t)) \subset N \backslash S^{\prime}$. containing the point $\varphi(\gamma(0))$. Then the initially given local isometry $\varphi$ analytically continues to the isometry of some neighborhood of the curve $\gamma(t), 0 \leq t \leq 1$, to some neighborhood of the curve $\beta(t), 0 \leq t \leq 1$, belonging to $N \backslash S^{\prime}$.

Let $M$ be a regular pseudocomplete Riemannian analytic manifold whose Lie algebra of all vector fields has no center, $S$ be the set of fixed points of all local isometries of the manifold $M$ preserving orientation and Killing vector fields, $M_{0}$ be a quasicomplete manifold locally isometric to $M, \widetilde{M}_{0}$ be simply connected covering of the manifold $M_{0}$. Then there are analytic locally isometric coverings $\widetilde{M}_{0} \rightarrow M \backslash S \rightarrow M_{0}$.

For an arbitrary oriented Riemannian analytic manifold $M$, we denote by $Z(M)$ the pseudogroup consisting of all local isometries of the manifold $M$ that preserve the orientation and all Killing vector fields. Let us consider the factor manifold $K_{M}$ of the manifold $M \backslash S$ by the pseudogroup $Z(M)$. We define the union of the manifolds $K_{M}$ and $K_{N}$ by gluing them over the set $K_{M \cap N}$. By the intersection 
$M \cap N$ we mean the identification of maximal subsets to which the initially given local isometry between the simply connected coverings $\widetilde{M}$ and $\widetilde{N}$ of the manifolds $M$ and $N$ is extended.

Let us consider the distribution $3^{\perp}$ on a manifold $M \backslash S$, consisting of vectors perpendicular to the center $z$ of the Lie algebra $g$ of all Killing vector fields.

Theorem 7. Let $M$ be pseudocomplete analytic Riemannian manifold, $3^{\perp}$ the distribution of tangent vectors perpendicular to the center $z$ of the algebra of all Killing vector fields, $S$ be the set of fixed points of local isometries preserving the orientation and all Killing vector fields. If $3^{\perp}$ is involutive, then the simply connected covering $\widetilde{M \backslash S}$ of the manifold $M \backslash S$ is isometric to the direct product of the Euclidean space and the simply connected covering $\widetilde{K}$ of the totally geodesic submanifold $K \subset M$ tangent to $z^{\perp} . \widetilde{M \backslash S} \approx \mathbb{R}^{k} \times \widetilde{K}$.

Proof. Since the distributions $z$ and $z^{\perp}$ are involutive, some neighborhood $U$ of the marked point $p \in M$ has the form $U=V \times W$ where $V$ is an open subset of the integral submanifold of the distribution $z$ and $W$ is an open subset of the integral submanifold of the distribution $3^{\perp}$. Let $x^{1} ; x^{2} ; \ldots ; x^{k}$ be coordinates on $V$, and $y^{1} ; y^{2} ; \ldots ; y^{m}$ be coordinates on $W$. Then in coordinates $x^{1} ; x^{2} ; \ldots ; x^{k} ; y^{1} ; y^{2} ; \ldots ; y^{m}$ components $g_{i j}$ do not depend on $x^{1} ; x^{2} ; \ldots ; x^{k}$, and since the submanifolds $\mathrm{V}$ and $\mathrm{W}$ are perpendicular, the components $d x^{i} d y^{j}$ are equal to 0 . Therefore, the metric on is $d s^{2}=d s_{1}^{2}(y)+f_{i j}(y) d x^{i} d x^{j}$. Since the pseudocomplete manifold $M \backslash S$ is nonextendable, it contains complete integral submanifold of the distribution $z$ that is direct products of the Euclidean space and the torus $\mathbb{R}^{s} \times T^{l}$. Therefore $M \backslash S$ is a fiber bundle over $K^{\prime} \subset K$ with fibers $\mathbb{R}^{s} \times T^{l}$. Since the distribution $\jmath^{\perp}$ is involutive, this fiber bundle contains the section $K^{\prime}$, and therefore it is trivial $M \backslash S=\mathbb{R}^{s} \times T^{l} \times K^{\prime}$. Since $M$ is not extendable then $K^{\prime}=K$. Therefore the simply connected covering of the manifold is isometric to the direct product of simply connected spaces $\widetilde{M \backslash S} \approx \mathbb{R}^{k} \times \widetilde{K}$.

Corollary. 3 Let us consider a Riemannian analytic manifold $M^{\prime}$ of dimension $n$ whose Lie algebra $\mathfrak{g}$ is commutative, that is, coincides with its center 3 and $\operatorname{dimg}=\operatorname{dim} z=n-1$. Then there are at most two pseudocomplete manifolds locally isometric to $M^{\prime}$.

Proof. Since $\operatorname{codim} \mathfrak{z}=1, \operatorname{dim}_{\mathfrak{3}}{ }^{\perp}=1$ and $\mathfrak{z}^{\perp}$ is involutive. By Theorem 5 , for a pseudocomplete manifold $M$ locally isometric to the manifold $M^{\prime}$, the decomposition $M \backslash S=\mathbb{R}^{s} \times T^{l} \times K$ holds. A completely geodesic submanifold $K$ is isometric to a line $\mathbb{R}$ or a circle $S^{1}$ or a ray $(a ; \infty)$ or an interval $(a ; b)$. Let us consider the factor set $\bar{K}=M / Z(M)$. If $K=\mathbb{R}$ or $K=S^{1}$ then $\bar{K}=K$. If $K=(a ; \infty)$, then $\bar{K}=[a ; \infty)$ or $\bar{K}=K=(a ; \infty)$. If $K=(a ; b)$ then $\bar{K}=[a ; b)$ or $\bar{K}=(a ; b]$ or $\bar{K}=[a ; b]$ or $\bar{K}=K=(a ; b)$.

If $K=\mathbb{R}$ or $K=S^{1}$ then the corresponding germ of an analytic Riemannian manifold has a unique extension to a pseudocomplete manifold, and this manifold is isometric to a Euclidean space. The extension of a germ to a pseudocomplete manifold will be unique in the case $S=\varnothing$ that is $\bar{K}=K$.

Let $K=(a ; \infty)$ and $\bar{K}=[a ; \infty)$. Then the points of the subset $S \subset M$ are mapped under the factorization $\bar{K}=M / Z(M)$ to the point $a \in \bar{K}$. A point $x \in S$ is a singular point of some field $X \in z$, $X(x)=0$ and any isometry $\varphi$ from $M$ into itself such that $\varphi(x)=x$ has the form $\varphi=\operatorname{Exp} Y, Y \in z$. Let us consider a subalgebra $z_{0} \subset z$ consisting of Killing vector fields $X \in \beta$ that vanish at the point $x$, $X(x)=0$. Then 30 generates the isometry group of some ball $B$ extending analytically to the isometry group of the manifold $M$ and isomorphic to the quotient group of the group $3_{0}=\mathbb{R}^{s}$ by some lattice $\Gamma$ acting on the manifold $M$. Then $M$ is a complete manifold isometric to the space $\mathbb{R}^{s} \times T^{l}$. A similar construction is applicable to the case when $K=(a ; b)$ and $\bar{K}=[a ; b)$ or $\bar{K}=(a ; b]$ that is, when $\bar{K}$ is obtained from by adding one point $a$ or $b$. In this case, the pseudocomplete manifold is also unique and isometric to the manifold $\mathbb{R}^{s} \times T^{l} \times \bar{K}$ however this manifold is no longer complete.

Finally, let us consider the case $K=(a ; b), \bar{K}=[a ; b]$. It means that $\bar{K}$ is obtained from $K$ by adding two points $a$ and $b$. Consider a pseudocomplete manifold $M_{1}$ and points of the set $S_{1} \subset M_{1}$ projecting to a point $a \in \bar{K}$. Then, as in the consideration of the previous cases, consider the variety $M_{1}^{\prime}$ obtained by joining the set $S_{1}$ to the quotient variety of the manifold $M \backslash S$ by some lattice $\Gamma_{1} \subset z=\mathbb{R}^{n-1}$ so that $M_{1}^{\prime}=\mathbb{R}^{s} \times T^{l} \times \overline{K_{1}}$ where $\overline{K_{1}}=[a ; b)$. Similarly, consider the pseudocomplete manifold $M_{2}$ and points 
of the set $S_{2} \subset M_{2}$ projecting to the point $b \in \bar{K}$. The manifold $M_{2}$ is obtained by joining the set $S_{2}$ to the quotient manifold of the manifold $M \backslash S$ by some lattice $\Gamma_{2} \subset z=\mathbb{R}^{n-1}$ so that $M_{2}^{\prime}=\mathbb{R}^{s} \times T^{l} \times \overline{K_{2}}$ where $\overline{K_{2}}=(a ; b]$. If the lattices $\Gamma_{1}$ and $\Gamma_{2}$ do not coincide, then the manifolds $M_{1}=M_{1}^{\prime}$ and $M_{2}=M_{2}^{\prime}$ are two different pseudocomplete manifolds. If the lattices $\Gamma_{1}$ and $\Gamma_{2}$ coincide, then the manifolds $M_{1}$ and $M_{2}$ are isometric and define the complete manifold $M=M_{1}=M_{2}$.

Let us turn to the description of pseudocomplete manifolds of small dimensions.

Let us consider a germ $\mathcal{A}$ of a two-dimensional Riemannian real analytic manifold. The Lie algebra $g$ of Killing vector fields of a two-dimensional manifold has dimension at most 3 . If $\operatorname{dimg}=3$ then the germ $\mathcal{A}$ is a germ of a manifold of constant curvature and extends to a complete manifold that is a sphere, a plane, or a hyperbolic plane. If $\operatorname{dimg}=2$ then the germ $\mathcal{A}$ is the germ of the left-invariant Riemannian metric on the two-dimensional Lie group, which is the extension of this germ to the complete manifold. The case $\operatorname{dimg}=1$ is described in the corollary to Theorem 5 just proved.

Let us consider completely inhomogeneous two-dimensional analytic Riemannian manifolds. The factor manifold $K$, constructed earlier as the union of all factor varieties locally isometric to each other, by the pseudogroup of all local isometries that preserve all Killing vector fields and orientation, is nothing more than a quasicomplete variety. Let us consider the set $\bar{K}=K \cup T$ obtained by adding to the manifold the images of points under the quotient mappings $\pi: M_{\alpha} \rightarrow M_{\alpha} / Z\left(M_{\alpha}\right)=\bar{K}_{\alpha} \subset \bar{K}$ defined on all possible analytic extensions $M_{\alpha}$ of the germ. Then the subset $T \subset \bar{K}$ consists of isolated points, and on $\bar{K}$ one can introduce the structure of an analytic manifold. Let us consider a point $z_{0} \in T \subset \bar{K}$. Then there is a sufficiently small ball $U_{0}$ centered at the point $x_{0} \in U_{0}$ such that the quotient mapping $\pi: U_{0} \rightarrow \bar{K}$ is a factorization of the ball $U_{0}$ by a finite group of rotations centered at $x_{0} \in U_{0}, \pi\left(x_{0}\right)=z_{0}$. Let $\mathrm{z}$ be a complex coordinate on $U_{0}$ such that the point $x_{0}$ has coordinate 0 . Then the mapping has the form $z \rightarrow w=z^{m}$ and the metric on the set $V_{0}=\pi\left(U_{0}\right) \subset \bar{K}$ is $d s^{2}=|w|^{-2(m-1) / m} d s_{1}^{2}(w ; \bar{w})$ where $d s_{1}^{2}(w ; \bar{w})$ is an analytic Riemannian metric on the ball $V_{0} \subset \bar{K}$.

We denote by $\widetilde{K}$ the simply connected covering of the set $\bar{K}$. Then the preimage $\widetilde{T} \subset \widetilde{K}$ of the set $T \subset \bar{K}$ is the discrete set of points $a_{i} \in \widetilde{K}$. An analytic Riemannian metric is uniquely defined on $\widetilde{K} \backslash \widetilde{T}$ so that the covering is locally isometric. Then the metric in the neighborhoods of the points $a_{i}$ has the form $d s^{2}=|w|^{-2(m-1) / m} d s_{1}^{2}(w ; \bar{w})$, if the complex coordinate $w$ is chosen so that point $a_{i}$ has coordinate 0 . A simply connected maifold $\widetilde{K}$ is diffeomorphic to a complex plane, circle, or sphere.

Let us consider the case when $\widetilde{K}$ is identified with the complex plane $\mathbb{C}$. Then there exists a function $f(z)$ holomorphic on $\widetilde{K} \backslash \widetilde{T}$ with branch points $a_{i}$ of order $m_{i}$. This function $f(z)$ is called the Weierstrass function. $f(z)=\prod_{i=1}^{\infty} \sqrt[m_{i}]{1-\frac{z}{a_{i}}} e^{\frac{1}{m_{i}}\left(\frac{z}{a_{i}}+\frac{1}{2}\left(\frac{z}{a_{i}}\right)^{2}+\ldots+\frac{1}{p_{i}}\left(\frac{z}{a_{i}}\right)^{p_{i}}\right)}$, where the numbers $p_{i} \in \mathbb{N}$ are such that $\forall z \in \mathbb{C}$ the series $\sum_{i=1}^{\infty}\left(\frac{z}{a_{i}}\right)^{p_{i}}$ converges. Let us consider the Riemann surface $M$ of the function $f(z)$. The surface $M$ covers the complex plane $\mathbb{C}$ so that the covering map $\pi: M \rightarrow \mathbb{C}$ has a branching of order $m_{i}$ over the points $a_{i} \in \mathbb{C}$ and is not $\mathrm{r}$ branching at other points. We define a Riemannian metric on $M g(X ; Y)=g\left(\pi_{*} X ; \pi_{*} Y\right)$ where $X, Y \in T_{x} M, \pi_{*} X, \pi_{*} Y \in T_{\pi(x)} K$. This metric has no singularities at the points $x_{i} \in M$ such that $\pi\left(x_{i}\right)=a_{i}$. It is easy to prove that for any simply connected manifold $N$ locally isometric to $M$, any local isometry $\varphi$ from $N$ to $M$ can be analytically extended to a locally isometric map $\varphi: N \rightarrow M$. So $M$ is the only analytic extension of this germ to a pseudocomplete manifold.

In case then $\widetilde{K}$ is a circle a unique analytic extension of this germ to a pseudocomplete manifold can be constructed similarly to the case of the complex plane. This manifold is also a Riemann surface of a holomorphic function $f(z)$ on $\widetilde{K}$ with branching of order $m_{i}$ over the points $a_{i} \in \widetilde{T} \subset \widetilde{K}$. $f(z)=\prod_{i=1}^{\infty} \sqrt[m_{i}]{\frac{z-a_{i}}{z-\alpha_{i}}} \sum_{k=1}^{q_{i}} \frac{\left(a_{i}-\alpha_{i}\right)^{k}}{k\left(z-\alpha_{i}\right)^{k}}$ where $\alpha_{i}$ is the point on the boundary of the circle closest to $a_{i}$ and the numbers $q_{i} \in \mathbb{N}$ are such that $\left|\ln \frac{z-a_{i}}{z-\alpha_{i}}+\sum_{k=1}^{q_{i}} \frac{\left(a_{i}-\alpha_{i}\right)^{k}}{k\left(z-\alpha_{i}\right)^{k}}\right|<\frac{1}{2^{i}}$. 
Let us consider the case when $\widetilde{K}$ is a sphere. Here the set $\widetilde{T} \subset \widetilde{K}$ consists of a finite number of points $\alpha_{0}, \alpha_{1}, \ldots, \alpha_{l}$ at each of which the metric has a singularity of the form $d s^{2}=|w|^{-2(m-1) / m} d s_{1}^{2}(w ; \bar{w})$. The function $f(z)=\prod_{i=1}^{l} \sqrt[m_{i}]{\frac{z-a_{i}}{z-\alpha_{0}}}$ on the sphere has branching of order $m_{i}$ at the points $a_{i}, \mathrm{i}=1 ; 2 ; \ldots ; 1$, and branching of order $m$ at the point $\alpha_{0}$. As above, consider the Riemann surface $M$ of the function $f(z)$. The covering map $\pi: M \rightarrow \widetilde{K}=S^{2}$ is a covering over $\widetilde{K} \backslash \widetilde{T}$ and has branching of order $m_{i}$ over the points $a_{i} \in \widetilde{T} \subset \widetilde{K}, i=1 ; 2 ; \ldots ; l$, and branching of some order $m$ into point $\alpha_{0}$. Then the metric on $M$ induced by the metric on $\widetilde{K}$ and the covering map $\pi$ has no singularity at the points $\pi^{-1}\left(a_{i}\right)$, but the case where $m \neq m_{0}$ has a singularity at the point $\pi^{-1}\left(a_{0}\right)$. The resulting manifold is a regular pseudocomplete manifold. Instead of the point $a_{0} \in \widetilde{T} \subset \widetilde{K}$, we can take any other point $a_{j} \in \widetilde{T} \subset \widetilde{K}$ and construct another regular pseudocomplete manifold in the way described above. Thus, we obtain all analytic extensions to the pseudocomplete manifold of the given germ.

Let us pass to the description of three-dimensional pseudo-complete manifolds. As before, we will denote by $z$ the center of the Lie algebra $g$ of all Killing vector fields on the manifolds under consideration. If $\operatorname{dim}_{\mathfrak{3}}=3$ then the germ of a Riemannian manifold is homogeneous and, according to a result of Mostov, [4], it can be extended to a homogeneous manifold. If $\operatorname{dim} \mathfrak{z}=2$ then, according to the proved corollary to Theorem 5 , there are at most two analytic extensions of a given germ to a regular pseudocomplete variety. The case when the algebra $g$ has no center, $\operatorname{dim} \mathfrak{z}=0$, was analyzed in the proof of Theorem 4.

Let us consider the case when $\operatorname{dim}_{\mathfrak{z}}=1$. First, consider the case when $\widetilde{K}$ is diffeomorphic to the plane. Let us consider the manifold $M_{0} \approx \widetilde{K} \times z$. Let $U_{0}$ be a small ball equipped with an initially given Riemannian metric on $V_{0}=U_{0} / Z\left(U_{0}\right) \subset \widetilde{K}$. We extend the metric defined on $U_{0}$ to the manifold $V_{0} \times \mathfrak{z}$. Let $x^{1} ; x^{2} ; x^{3}$ be coordinates on $V_{0} \times 3$ such that $x^{1} ; x^{2}$ are coordinates on $V_{0}$ and $x^{3}$ is a coordinate on 3 . The components of the metric tensor $g_{i j}\left(x^{1} ; x^{2}\right)$ are independent of $x^{3}$. The functions $g_{i j}\left(x^{1} ; x^{2}\right)$ extend analytically along any curve on $\widetilde{K}$ and define a metric on $M_{0} \approx \widetilde{K} \times \mathfrak{z}$. Then $M_{0} / Z\left(M_{0}\right)=K$, hence $Z\left(M_{0}\right)=K \times \Gamma$ where $\Gamma$ is the group of covering $\widetilde{K} \rightarrow K$. Then, for a regular pseudocomplete manifold $M$ the manifold $M \backslash S=K \times 3 / \Gamma_{0}$ where $\Gamma_{0}$ is a discrete subgroup of the group $\jmath$.

Let us now consider the case when the quotient variety $K$ is diffeomorphic to a sphere. Let us split $K$ into a union of two open disks $K=K_{1} \cup K_{2}$. We construct, as above, the Riemannian manifolds $M_{1}=K_{1} \times \mathbb{R}$ and $M_{2}=K_{2} \times \mathbb{R}$ which are analytic extensions of the originally given germ, whose'submanifolds $\mathbb{R}$ are integral curves of the vector field $X \in \mathfrak{z}$. Local isometries $f$ from $M_{1}$ to $M_{2}$ extend along any curve on $\left(K_{1} \cap K_{2}\right) \times \mathbb{R}$. If such an extension is unique, we get a complete manifold $M \approx S^{2} \times \mathbb{R}$ which is an extension of a given germ. Suppose now that there exists a closed curve $\gamma(t), 0 \leq t \leq 1$, on $\left(K_{1} \cap K_{2}\right) \times \mathbb{R}$, the extension of the isometry $f$ along which is ambiguous, $f(\gamma(0))=y_{1} \neq y_{2}=f(\gamma(1))$. Let $x^{1} ; x^{2} ; x^{3}$ are coordinates on $M_{1}$ such that $x^{1} ; x^{2}$ form coordinates on $K_{1}$ and $x^{3}$ is a coordinate on $\mathbb{R}$, and $y^{1} ; y^{2} ; y^{3}$ are coordinates on $M_{2}$ such that $y^{1} ; y^{2}$ form coordinates on $K_{2}$ and $y^{3}$ is a coordinate on $\mathbb{R}$. Since $x^{3}$ and $y^{3}$ are coordinates on the Lie algebra $z$ the isometry $f$ in coordinates $x^{1} ; x^{2} ; x^{3} ; y^{1} ; y^{2} ; y^{3}$ have the form $y^{1}=y^{1}\left(x^{1} ; x^{2}\right), y^{2}=y^{2}\left(x^{1} ; x^{2}\right), y^{3}=x^{3}+f\left(x^{1} ; x^{2}\right)$ where the functions $y^{1} ; y^{2}$ are the transition functions from the map on $K_{1}$ to the map on $K_{2}$ on sphere and therefore are unambiguous. The function $f\left(x^{1} ; x^{2}\right)$ continues ambiguously along the closed curve $\delta(t), 0 \leq t \leq 1$, to $K_{1} \cap K_{2}$. Let $f(\delta(1))-f(\delta(0))=a \in \mathbb{R}$. Let's consider the circle $S^{1}=\mathbb{R} / \mathbb{Z}$. Then the extension of the function $f$ along the curve $\delta$ will be unique if we assume that $f$ takes values not on the line $\mathbb{R}$, but on the circle $S^{1}$. Then, there will also be a unique extension of the function $f$ along the curves $\delta^{n}, n \in \mathbb{Z}$. But, since any curve on $K_{1} \cap K_{2}$ is homotopic to the curve $\delta^{n}$ the extension of the function $f: U_{0} \rightarrow S^{1}$ along all possible curves is unique on $K_{1} \cap K_{2}$. In this case, the function $f$ is the transition function of the bundle into circles over the sphere $S^{2}$ and we have compact lens spaces as analytic extensions of a given germ. 


\section{Locally Homogeneous Pseudo-Riemannian Manifolds, the Algebra of Killing Vector Fields Which Has a Nontrivial Center}

Let us investigate the case when the algebra $g$ has a nonzero center $z$ and indicates the properties of the algebras $\mathfrak{g}, \mathfrak{h}$, and $\mathfrak{z}$ that ensure the closeness of the subgroup $H$ in $G$.

Let us define a local group of local isometries. Let us consider an arbitrary Riemannian analytic manifold $\mathfrak{g}$, a Lie algebra $G$ consisting of all Killing vector fields on it, and a Lie group $G$ with Lie algebra g. By a local group (chunk of a group) we mean a small neighborhood of the identity of the group $U \subset G$. It consists of local isometries of the manifold $M$. Consider a normal ball $B_{2 \delta}$ of radius $2 \delta$ centered at $p \in M$. The neighborhood of the identity $U$ in the group $G$ consists of elements $g \subset G$ that define isometries from the ball $B_{\delta}$ of radius $\delta$ centered at a marked point $p \in M$ to the ball $B_{2 \delta}$ of radius $2 \delta$ centered at $p \in M$. Note that $H \subset U$. The Lie algebra $g$ as a rule does not generate the isometry group of the manifold $M$ but generates the pseudogroup $U$ of local isometries. The orbit of the local group of local isometries of the manifold $M$ is a locally homogeneous manifold $N$. Note also that the subgroup $H$ generated by the stationary subalgebra $\mathfrak{h}$ forms the isometry group of the ball $B_{\delta}$ centered at a marked point of $M$.

Let us study some properties of the local group of local isometries from the point of view of abstract transformation groups. Let us consider a local group $U \subset G$ as a subgroup of the group of local diffeomorphisms of a manifold $M$ with a marked point $p, G \subset$ DiffM. We call an element $\tilde{n} \in G \subset \operatorname{DiffM}$ right multiplication if there exists an element $n \in G$ such that for all $x \in M$ such that $x=g(p), \widetilde{n}(x)=g n(p)$. Since $\forall h \in H \cdot g h(p)=g(p)=x$ then $\widetilde{n}(x)=g n(p)=g h n(p)$. Therefore $n(p)=h n(p) \Rightarrow p=n^{-1} h n(p) \Rightarrow n^{-1} h n \in H$. Thus, right multiplication by an element $n$ is well defined if $\forall h \in H \exists h_{1} \in H$ is such that for any local isometry $g \in G$ the equality $g h n=g n h_{1}$ holds. In other words, $n$ belongs to the normalizer $N(H)$ of the group $H$ in $G$. Let $N$ denote the local group consisting of elements $n \in G$ right multiplication by which in the group $G$ generate local isometries of the manifold $M$ and by its Lie algebra. Then $\mathfrak{h} \triangleleft \mathfrak{n} \subset \mathfrak{g}$. Note that the multiplications themselves on the right, that is, the elements $\tilde{n}$ as well as the elements of the center $Z$ of the local group $G$ belong to $N$. Let $V$ denote the orbit of the marked point $p$ of the local group $N$ on $M$. The adjoint action of the elements $n \in N, g \mapsto n^{-1} g n$ defines local isometries on $V$.

Let us find a subgroup $G_{0} \subset G$ consisting of "multiplications on the left." Consider a mapping $f$ from a group $G$ defined as the group of transformations of a set $G$ into itself, defined by the formula $f(g)=g(e)=g e$ where $e$ is the identity local isometry. Then, since $\widetilde{n}(e)=\widetilde{n} e=e n=n$ we will assume that $f(\widetilde{n})=n$. Strictly speaking $f(\widetilde{n})$ is the coset $n h$ but all elements $n h, h \in H$ define the same local isometry of the manifold $M$. On the set $f(G)$ we define the multiplication $g_{1} g_{2}=g_{1}(e) g_{2}(e)$. The multiplication thus defined turns $f(G)$ into a subgroup $G_{0} \subset G$. Left multiplications $g \in G_{0}$ are supplemented by right multiplications $\tilde{n}$ that is, any element $g \subset G \subset \operatorname{DiffG}, g(x)=g x \forall x \in G$ can be represented as $g=g_{0} \widetilde{n}, g_{0} \widetilde{n}(x)=g_{0} x n \forall x \in G$ Since the elements $n, n h$ and $n \in N, h \in H$, using multiplications on the right in the group $G$ define the same local isometry on $M$ the group of "multiplications on the right" is identified with the factor group $\widetilde{N}=N / H$ and the Lie algebra of this group is identified with the factor algebra $\mathfrak{n} / \mathfrak{h}$. Therefore $G=G_{0} \widetilde{N}, \mathfrak{g}=\mathfrak{g}_{0}+\widetilde{\mathfrak{n}}$.

Let us consider $V \subset B_{2 \delta}$ that the orbit of the marked point $\mathrm{p}$ of the local isometry group $\widetilde{N}$. The group of "left multiplications" $N \subset G_{0}$ (more precisely, its neighborhood of unity) acts on $V$; moreover $n$, $h n$ and $n h, n \in N, h \in H$ define the same local isometry on $V$ by means of left multiplications in the group $N$ then the group of "left multiplications" in the group $N$ is also identified with the quotient group $\widetilde{N}=N / H$, and the Lie algebra $\widetilde{\pi}$ of this group is identified with the quotient algebra $\mathfrak{n} / \mathfrak{h}$.

Thus, the inner automorphisms of the group $\widetilde{N}$ are isometries of $V$ and generate the adjoint representation of the group $\widetilde{N}$ in the algebra and form the adjoint group Int $(\widetilde{n})$ of the algebra $\widetilde{n}$. Since $\widetilde{N}=N / H$ acts on $V$ transitively, then $\widetilde{\pi}$ can be identified with the tangent space $T_{p} V$ and Int $(\widetilde{\mathfrak{n}})$ is a closed subgroup of the group $G L\left(T_{p} V\right)$ of linear transformations of the space $T_{p} V$. But, since Int $(\widetilde{\mathfrak{n}})$ 
preserves a nondegenerate positive definite Riemannian form on $T_{p} V$ then Int $(\widetilde{\pi})$ is a closed subgroup of the compact group of orthogonal transformations $S O\left(T_{p} V\right)$ and therefore is compact.

The group Int $(\widetilde{n})$ is isomorphic to the group $\widetilde{N} / Z(\widetilde{N})$ where $Z(\widetilde{N})$ is the center of the group $\widetilde{N}$. Therefore, the group $\widetilde{N} / Z(\widetilde{N})$ is compact and the Lie algebra $\widetilde{\pi}$ is a compact algebra [1]. Therefore $\widetilde{\pi}$ is decomposed into the direct sum of its center $z$ and the commutant $[\tilde{n} ; \tilde{n}], \widetilde{n}=z \oplus[\tilde{n} ; \tilde{\mathfrak{n}}]$. Since all "right multiplications" commute with all "left multiplications", then 3 coincides with the center of the entire algebra of Killing vector fields $\mathfrak{g}$. Thus there is a direct sum decomposition $\mathfrak{g}=\mathfrak{g}_{0} \oplus \mathfrak{z} \oplus[\mathfrak{n} ; \tilde{\mathfrak{n}}$. $[\tilde{\mathfrak{n}} ; \tilde{\mathfrak{n}}]$ generates "pure right multiplications" that do not coincide with "left multiplications.

Theorem 8. Let $\mathrm{g}$ be the Lie algebra of all Killing vector fields on a locally homogeneous pseudo-Riemannian analytic manifold $M, \mathfrak{h}$ be its stationary subalgebra, 3 be the center of $\mathfrak{g}$. Let $G$ be a simply connected subgroup generated by the algebra $\mathfrak{g}$ and $H$ its subgroup generated by the subalgebra $\mathfrak{h}$. If $\mathfrak{h} \cap(\mathfrak{z}+[\mathfrak{g}, \mathfrak{g}])=\mathfrak{h} \cap[\mathfrak{g}, \mathfrak{g}]$ then $H$ is closed in $G$.

Proof. Suppose the opposite. Let us consider the closure $\bar{H}$ of the group $H$ in $G$ and the subalgebra $\overline{\mathfrak{h}} \subset \mathfrak{g}$ of the subgroup $\bar{H} \subset G$. The subalgebra $\mathfrak{h}$ is a normal subalgebra of the algebra $\overline{\mathfrak{h}}$ [3]. We will assume, passing if necessary to the conjugate group $g^{-1} H g$ which for a marked point $\in M X \in \mathfrak{h} \Leftrightarrow X(p)=0$. Let us consider the one-parameter subgroup $\bar{h}_{t} \in \bar{H}, \bar{h}_{t} \notin H$ defined by the vector field $\bar{X} \notin \mathfrak{h}, \bar{X} \notin \mathfrak{h}$. As proved in [3], there exists a torus $T$ in a simple compact subgroup $P \in G$ such that $H \cap T$ is an everywhere dense winding of the torus $T$. Therefore, we can assume that $\bar{h}_{t} \in T \subset P$. Then the Killing vector field of tangent vectors to the orbits of the local one-parameter group $\bar{h}_{t}$ belongs to the algebra $t$ of the group $T$ and, therefore $\bar{X} \in \mathfrak{t} \subset \mathfrak{p}$ where $\mathfrak{p}$ is the Lie algebra of the group $P$. Let us consider a neighborhood of unity $U$ in the group $G$ and the ball $B_{\delta}$ of radius $\delta$ centered at the marked point $p \in M$ such that all elements $g \in U$ of the group $G$ define local isometries from the ball $B_{\delta}$ to the ball $B_{2 \delta}$ of radius $2 \delta$ centered at $p \in M$. Note that $H \subset U$. Since the elements $\bar{h}_{t}$ belong to the closure $\bar{H}$ of $H$ in $G$ for each small $t$ the inner automorphism $x \mapsto \bar{h}_{t} x \bar{h}_{t}^{-1}$ of the group $G$ is the limit of the sequence of inner automorphisms $x \mapsto h_{n} x h_{n}^{-1}, h_{n} \in H$. For small $t$ and large $n$ these automorphisms define local isometries of the ball $h_{n} B_{\delta}$ into the ball $B_{2 \delta}$.

Inner automorphisms $x \mapsto h_{n} x h_{n}^{-1}, h_{n} \in H$ generating the same local isometries as multiplication by $h_{n}$ define isometries of the ball $B_{\delta}$. Since the elements $\bar{h}_{t}$ belong to the normalizer of the group $H$ the inner automorphisms $x \mapsto \bar{h}_{t} x \bar{h}_{t}^{-1}$ define maps on the ball $B_{\delta}$ that are limits of isometries, they also define the isometry of the ball $B_{\delta}$ into the ball $B_{\delta}$. Then, since the local isometry $x \mapsto \bar{h}_{t} x$ of the ball $B_{\delta}$ into the ball $B_{2 \delta}$ is defined for all sufficiently small $t$, the local isometry $x \mapsto x \bar{h}_{t}^{-1}=x \bar{h}_{-t}$ is also defined, and thus the local one-parameter group of isometries generated by multiplications on the right by the elements $\bar{h}_{-t}$ is defined.

All right multiplications commute with left multiplications that are with the elements of the group $G_{0}$. However they may not commute with each other. Let us prove that local isometry $\bar{h}_{-t}$ commutes with all right multiplications. To this end, we prove that the action of the element $\bar{h}_{-t}$ in the group of inner automorphisms of the group $G, g \mapsto \bar{h}_{t}^{-1} g \bar{h}_{t}$ defines the identity map on the orbit $V$ of the marked point $p$ of the group $\widetilde{N}$. Let us consider a sequence $h_{n} \in H$ converging to $\bar{h}_{t}^{-1}$. Since $N$ is a normal subgroup in $N$ then $n h_{n}=h_{n} n h_{n}^{\prime}$ where $h_{n}^{\prime} \in H$ then $n H=h_{n}^{-1} n h_{n} H$. Hence, the inner automorphisms $g \mapsto h_{n}^{-1} g h_{n}$ induce the identity map on $V$. Passing to the limit, we see that the inner automorphism $g \mapsto \bar{h}_{t}^{-1} g \bar{h}_{t}$ induces the identity map on $V$.

The vector field $Z$ of tangent vectors to the orbits of the local one-parameter group $z_{t}$ of multiplications on the right by $\bar{h}_{t}$ is a Killing vector field and belongs to the center of the algebra of all Killing vector fields on $M, Z \in \mathfrak{\jmath}$. It follows from the decomposition $\mathfrak{g}=\mathfrak{g}_{0} \oplus \mathfrak{\jmath} \oplus[\tilde{\mathfrak{n}} ; \widetilde{\mathfrak{n}}]$ that $Z \notin[\mathfrak{g} ; \mathfrak{g}]$. Therefore $X+Z \notin[\mathfrak{g} ; \mathfrak{g}]$ but $X+Z \in \mathfrak{h}$. This proves the theorem by contradiction. 
Since the vector field $X$ generating the local one-parameter group $\bar{h}_{t}^{-1}$ belongs to a compact subalgebra of the algebra $\mathfrak{g}$ then $X$ belongs to the commutator subalgebra $[\mathfrak{g} ; \mathfrak{g}]$ of the algebra $\mathfrak{g}$.

Theorem 9. Let $\mathfrak{g}$ be the Lie algebra of all Killing vector fields on a locally homogeneous pseudo-Riemannian analytic manifold $M, \mathfrak{h}$ is its stationary subalgebra, 3 is the center of $\mathfrak{g}$, and $\mathfrak{r}$ is its radical. Let $G$ be a simply connected subgroup generated by the algebra $\mathfrak{g}$ and $H$ its subgroup generated by the subalgebra $\mathfrak{h}$. Then, if for any semisimple algebra $\mathfrak{p} \subset \mathfrak{g}$ such that $\mathfrak{p}+\mathfrak{r}=\mathfrak{g}$ there is the equality $(\mathfrak{p}+\mathfrak{z}) \cap \mathfrak{h}=\mathfrak{p} \cap \mathfrak{h}$ then $H$ is closed in $G$.

Proof. Suppose the opposite and consider the closure $\bar{H}$ of the group $H$ in G. As in the proof of Theorem 5 , let us consider the one-parameter subgroup $z_{t}$ generated by right multiplication by elements of the one-parameter group of local isometries $\bar{h}_{t}$ in $G$. Let $\bar{X}$ be the Killing vector field of tangent vectors to orbits of the local one-parameter group of local isometries $\bar{h}_{t}^{-1}$ and $Z$ is the Killing vector field of the local one-parameter group of local isometries $z_{t}$.

Let $\mathfrak{p}$ be a semisimple subalgebra of the algebra $\mathfrak{g}$ containing the vector field $\bar{X}, \bar{X} \in \mathfrak{p} \subset \mathfrak{g}$. Let us prove that $Z+\bar{X} \in \mathfrak{h}$ and $Z+\bar{X} \notin \mathfrak{p}$. In a simply connected Lie group $G$, consider the radical $R$ (the subgroup corresponding to the subalgebra $r$ ) and the semisimple subgroup $P$ corresponding to the subalgebra $\mathfrak{p}$. Then $R$ is a normal subgroup in $G \mathrm{r}$ is a normal subalgebra in $\mathfrak{g}, R \cap P=e, \mathfrak{r} \cap \mathfrak{p}=0$ and the Levi-Maltsev decomposition $G=R P$ holds.

The group $G$ contains an open neighborhood of the identity (chunk of a group) acting as a local group of local isometries in a neighborhood of the marked point $p \in M$. Since $z_{t}$ belongs to the center of the group $G$, then $z_{t} \in R$ and since the subgroup $H$ is a normal divisor of the group $\bar{H}$, [3], then $\bar{h}_{t}^{-1} z_{t} H=\bar{h}_{t}^{-1} H \bar{h}_{t}=H$. Consequently, the local isometries $\bar{h}_{t}^{-1} z_{t}$ leave the point $p$ fixed and, therefore, belong to the stationary subgroup $H$. But, since $\bar{X} \in \mathfrak{p}$, and $Z \notin \mathfrak{p}$ then $(Z+\bar{X}) \notin \mathfrak{p}$. Since $(Z+\bar{X}) \in \mathfrak{h}$ this means that the statement $(\mathfrak{p}+\mathfrak{z}) \cap \mathfrak{h} \neq \mathfrak{p} \cap \mathfrak{h}$ holds for the chosen maximal semisimple algebra $\mathfrak{p}$. This proves the theorem by contradiction.

\section{Discussion}

Let us point to the questions related to the generalization of the completeness of a Riemannian analytic manifold that require a solution. First, we would like to give necessary and sufficient conditions for the closeness of a stationary subgroup of the group of local isometries on a Riemannian manifold. Moreover, these conditions must be expressed in local terms, that is, as properties of the Lie algebra of all Killing vector fields. In addition, it is suitable to develop a theory of generalized complete manifolds in the case of the existence of a nontrivial center in the Lie algebra of all Killing vector fields to give a definition of a quasicomplete manifold in the general case. It is also desirable to describe in more detail pseudocomplete manifolds in the general case and for specific Riemannian metrics. Theorems 8 and 9 give necessary and "almost sufficient" conditions for the closeness of a stationary subgroup. It would be nice to find necessary and sufficient conditions for the closeness of a stationary subgroup in a simply connected Lie group generated by all Killing vector fields.

Funding: This research received no external funding.

Conflicts of Interest: The author declares no conflict of interest.

\section{References}

1. Helgason, S. Differential Geometry, Lie Groups, and Symmetric Spaces; Academic Press, Inc.: Boston, MA, USA; Harcourt Brace Jovanovich: San Diego, CA, USA; New York, NY, USA, 1978.

2. Kobayashi, S.; Nomidzu, K. Foundations of Differential Geometry; Interscience Publisher: New York, NY, USA, 1969.

3. Smith, G.H. Analytic extension of Riemannian manifolds. Bull. Aust. Math. Soc. 1978, 18, 147-148. [CrossRef]

4. Maltsev, A.I. On the theory of Lie groups in the large. Mathem. Sb. 1945, 16, 163-190. 
5. Mostow, G.D. Extensibility of Local Lie Groups of Transformations and Groups on Surfaces. Ann. Math. 1950, 52, 606-636. [CrossRef]

6. Popov, V.A. On the Extendability of Locally Defined isometries of a Pseudo-Riemannian Manifolds. J. Math. Sci. 2016, 217, 624-627. [CrossRef]

7. Popov, V.A. On Closeness of Stationary Subgroup of Affine Transformation Groups. Lobachevskii J. Math. 2017, 38, 724-729. [CrossRef]

8. Popov, V.A. Extendability of Locally Defined Isometries of a Pseudo-Riemannian Manifold. J. Math. Sb. 1988, 135, 45-64. [CrossRef]

9. Popov, V.A. Analytic Extension of Locally Given Riemannian Manifolds. Math. Zam. 1984, 38, 559-570.

Publisher's Note: MDPI stays neutral with regard to jurisdictional claims in published maps and institutional affiliations.

(C) 2020 by the author. Licensee MDPI, Basel, Switzerland. This article is an open access article distributed under the terms and conditions of the Creative Commons Attribution (CC BY) license (http://creativecommons.org/licenses/by/4.0/). 